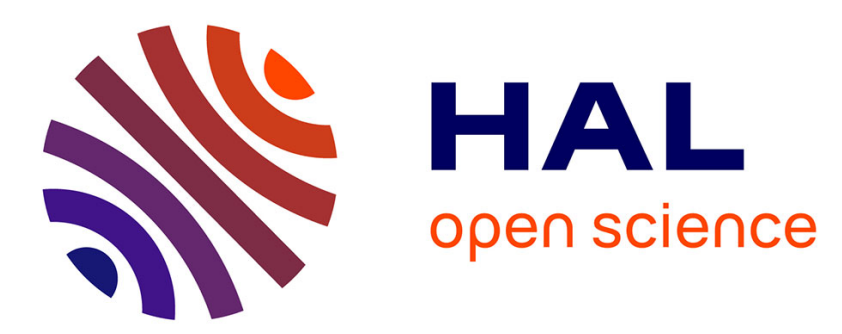

\title{
Appareillage et méthodologie d'étude des surfaces de GaAs en cours de croissance en épitaxie phase vapeur
}

\author{
J.B. Theeten, F. Hottier, H. Paradan
}

\section{To cite this version:}

J.B. Theeten, F. Hottier, H. Paradan. Appareillage et méthodologie d'étude des surfaces de GaAs en cours de croissance en épitaxie phase vapeur. Revue de Physique Appliquée, 1976, 11 (5), pp.587-595. 10.1051/rphysap:01976001105058700 . jpa-00244093

\section{HAL Id: jpa-00244093 https://hal.science/jpa-00244093}

Submitted on 1 Jan 1976

HAL is a multi-disciplinary open access archive for the deposit and dissemination of scientific research documents, whether they are published or not. The documents may come from teaching and research institutions in France or abroad, or from public or private research centers.
L'archive ouverte pluridisciplinaire HAL, est destinée au dépôt et à la diffusion de documents scientifiques de niveau recherche, publiés ou non, émanant des établissements d'enseignement et de recherche français ou étrangers, des laboratoires publics ou privés. 


\title{
APPAREILLAGE ET MÉTHODOLOGIE D'ÉTUDE DES SURFACES DE GaAs EN COURS DE CROISSANCE EN ÉPITAXIE PHASE VAPEUR (*)
}

\author{
J. B. THEETEN, F. HOTTIER et H. PARADAN
}

Laboratoires d'Electronique et de Physique appliquée

3, avenue Descartes, 94450 Limeil-Brévannes, France

(Reçu le 22 mars 1976, accepté le 17 mai 1976)

\begin{abstract}
Résumé. - La caractérisation, à l'échelle microscopique, de l'interface solide-vapeur au cours d'une réaction d'épitaxie en phase vapeur apporterait des éléments essentiels à la compréhension des mécanismes de croissance cristalline. On décrit ici un appareillage capable d'accéder à l'interface de croissance in vivo. Pour cela, un réacteur d'épitaxie vapeur a été aménagé pour permettre des mesures d'ellipsométrie. Pour compléter les informations obtenues, un système de sas permet un transfert direct dans un bâti ultra-vide où on examine la surface en diffraction électronıque LEED et RHEED et en spectrométrie AUGER. On illustre le fonctionnement du système sur le cas de couches épitaxiales de $\mathrm{GaAs}(100)$ préparées dans le système $\mathrm{H}_{2}-\mathrm{GaAs}-\mathrm{AsCl}_{3}$.

Abstract. - The characterization, on an atomic scale, of the solid-vapour interface in a Chemical Vapour Deposition reaction is a key problem in the understanding of crystal growth mechanisms. An apparatus in described which allows in situ measurements of the interface during the growth. A conventional C.V.D. reactor has been adapted to allow ellipsometry measurements. To obtain further information a system has been set up that allows direct transfer from the reaction to an U. H. V. chamber with LEED, RHEED and AES facilities. The system is demonstrated for the case of (100) $\mathrm{GaAs}$ epitaxial layers obtained from the $\mathrm{H}_{2}-\mathrm{GaAs}-\mathrm{AsCl}_{3}$ system.
\end{abstract}

1. Introduction. - Le développement actuel de la microélectronique, dont la tendance est à demander des couches de plus en plus minces de silicium ou d'arséniure de gallium de qualités cristallines et électrıques aussi bonnes que possible, nécessite d'accroître nos connaissances fondamentales sur les mécanismes de croissance cristalline. Si on examine les données expérimentales connues à l'heure actuelle [1] la plupart consistent en des mesures macroscopiques (à l'échelle de $1 \mu \mathrm{m})$ a posteriori : microscopie optique donnant la morphologie et la vitesse de croissance, mesures électriques donnant les niveaux de dopage et d'impuretés, examen au microscope à balayage ou aux rayons $\mathrm{X}$. Des mesures plus microscopiques faites au microscope électronique (diffraction, transmission, répliques transfert) apportent des données précieuses sur les dislocations interfaciales. Mais il n'a pas été possible jusqu'à présent d'obtenir in situ des données à l'échelle atomique sur l'interface de croissance. Il est cependant clair que la connaissance de la nature chimique et de la structure cristalline de cet interface serait un apport essentiel. On pourrait notamment vérifier les modèles

(*) Ce travail a été fait avec l'aide de la D. G. R.S. T. sous contrat $\mathrm{n}^{\circ}$ 74.07.464. théoriques proposés à partir des observations macroscopiques. Un premier effort dans ce sens a été le développement récent de l'épitaxie par faisceaux moléculaires où on peut suivre à l'aide des techniques d'analyse de surface la croissance épitaxiale de GaAs. Les travaux de Arthur, Foxon et Joyce, Cho [2] Esaki et al. [3] ont largement démontré l'intérêt d'une telle méthode d'approche de la croissance cristalline.

Notre idée était d'essayer d'aller plus loin dans cette association entre les techniques d'analyse des surfaces et la croissance cristalline en envisageant un cas plus courant d'interface de croissance. Nous avons choisi l'épitaxie en phase vapeur de l'arséniure de gallium en raison d'une part des données déjà obtenues sur les surfaces de GaAs propres [4] ou en cours d'épitaxie par faisceaux moléculaires, d'autre part du grand nombre de données microscopiques disponibles sur ce système, données assez bien décrites par des modèles de thermodynamique statistique $[4,5]$.

Il nous fallait donc développer des techniques qui permettent d'accéder à la surface de GaAs in situ, c'est-à-dire dans les conditions de haute température $\left(800^{\circ} \mathrm{C}\right)$ et d'atmosphère réactive d'un réacteur de C. V. D. (Chemical Vapour Deposition). La seule particule de mesure possible dans cette ambiance est le photon. Pour des longueurs d'onde de 300 à 600 nm, 
la lumière est absorbée sur $1000 \AA$ environ dans GaAs. En outre, la mesure précise en ellipsométrie de la modification de la polarisation de la lumière réfléchie permet de détecter jusqu'à $1 / 100^{\mathrm{e}}$ de monocouche adsorbée sur un substrat. L'ellipsométrie est donc parfaitement adéquate pour les mesures in situ.

Cependant, l'interprétation des données ellipsométriques est délicate, en particulier dans ce cas où le substrat examiné est susceptible de modifications chimiques et cristallographiques. Il est donc nécessaire de compléter les analyses in situ par des analyses utilisant l'électron comme particule de mesure. Dans ce cas les données chimiques (par spectroscopie Auger) et structurales (par diffractions d'électrons LEED et RHEED) sont obtenues sans ambiguïté. Pour être utiles, ces mesures doivent être quasi in situ c'est-à-dire obtenues en perturbant le moins possible l'interface de croissance. On a donc réalisé un système permettant de transférer directement l'échantillon $\mathrm{du}$ réacteur C. V. D. dans un bati ultra-vide muni des systèmes LEED, RHEED et AUGER, sans remise à l'air. L'ellipsométrie permet d'ailleurs de suivre ce transfert de l'ambiance haute pression-haute température à l'ambiance ultra vide-température ambiante et d'apprécier les modifications subies par l'interface au cours du transfert. Après une description de l'appareillage et des méthodes de mesure, nous donnerons quelques résultats typiques obtenus.

2. Description de l'appareillage. - Une vue générale de l'appareillage est donnée sur la figure 1. La partie inédite consiste en la réalisation d'une liaison entre un réacteur C. V. D. et un bâti ultra-vide (pression dans la gamme des $10^{-10}$ torr) aussi conventionnels que possible.

2.1 RÉACTEUR D'ÉPITAXIE. - On utilise pour l'épitaxie le système $\mathrm{GaAs}-\mathrm{H}_{2}-\mathrm{AsCl}_{3}$ c'est-à-dire une source solide de GaAs portée à une température $T_{\mathrm{S}}$ de $800^{\circ} \mathrm{C}$ environ, sur laquelle arrive un flux de $\mathrm{AsCl}_{3}$ dilué dans $\mathbf{H}_{2}$. Le trichlorure d'arsenic est décomposé à la température $T_{\mathrm{S}}$ en présence de $\mathrm{H}_{2}$, suivant la réaction

$$
\mathrm{AsCl}_{3}+\frac{3}{2} \mathrm{H}_{2} \rightarrow \frac{1}{4} \mathrm{As}_{4}+3 \mathrm{HCl} \text {. }
$$

Le transport de GaAs de la source au substrat se fait par l'intermédiaire de $\mathrm{HCl}$ fourni par la réaction (1) en utilisant la réaction $[6,7]$

$\mathrm{GaAs}_{\text {solide }}+\mathrm{HCl}_{\mathrm{gaz}} \rightleftarrows \mathrm{GaCl}_{\mathrm{gaz}}+\frac{1}{4} \mathrm{As}_{4 \mathrm{gaz}}+\frac{1}{2} \mathrm{H}_{2 \mathrm{gaz}}$.

A la température $T_{\mathrm{S}}$ de la source, la réaction (2) est dans le sens de l'attaque du GaAs, à la température $T_{\mathrm{E}}$ de l'échantillon (2) est dans le sens de la formation de $\mathrm{GaAs}$ solide. En toute rigueur la réaction (1) apporte un excès de $\mathrm{As}_{4}$. Celui-ci peut servir à la fois à resaturer la source en As et au dépôt sur l'échantillon. Les conditions choisies pour les températures, les pressions partielles et les flux gazeux sont celles utilisées habituellement. Elles permettent d'établir trois régimes sur lesquels nous reviendrons au paragraphe 3 :

- régime de montée en température de l'échantillon $T_{\mathrm{S}}=800^{\circ} \mathrm{C}, T_{\mathrm{E}}=750^{\circ} \mathrm{C}$, flux d'hydrogène vecteur $=17 \mathrm{l} / \mathrm{h}$, dans un réacteur en quartz de diamètre $40 \mathrm{~mm}$;

- régime de décapage, mêmes températures, même flux de $\mathrm{H}_{2}$ vecteur, pression de $\mathrm{AsCl}_{3}=10$ torr amené par un flux de $\mathrm{H}_{2}$ de $6 \mathrm{l} / \mathrm{h}$ directement sur l'échantillon sans passer sur la source ;

- régime de croissance, mêmes températures, flux de $\mathrm{H}_{2}$ vecteur : $7 \mathrm{l} / \mathrm{h}$, pression de $\mathrm{AsCl}_{3}=10$ torr amené sur la source par un flux de $\mathrm{H}_{2}$ de $11 \mathrm{l} / \mathrm{h}$. Dans un tel régime, on obtient des vitesses de croissance de l'ordre de $20 \mu \mathrm{m} / \mathrm{h}$.

Les températures de source et d'échantillon sont obtenues à l'aide d'un four électrique à 2 zones disposé autour du tube de quartz. Le four est muni de 2 ouvertures de $10 \mathrm{~mm}$ de diamètre au niveau de l'échantillon

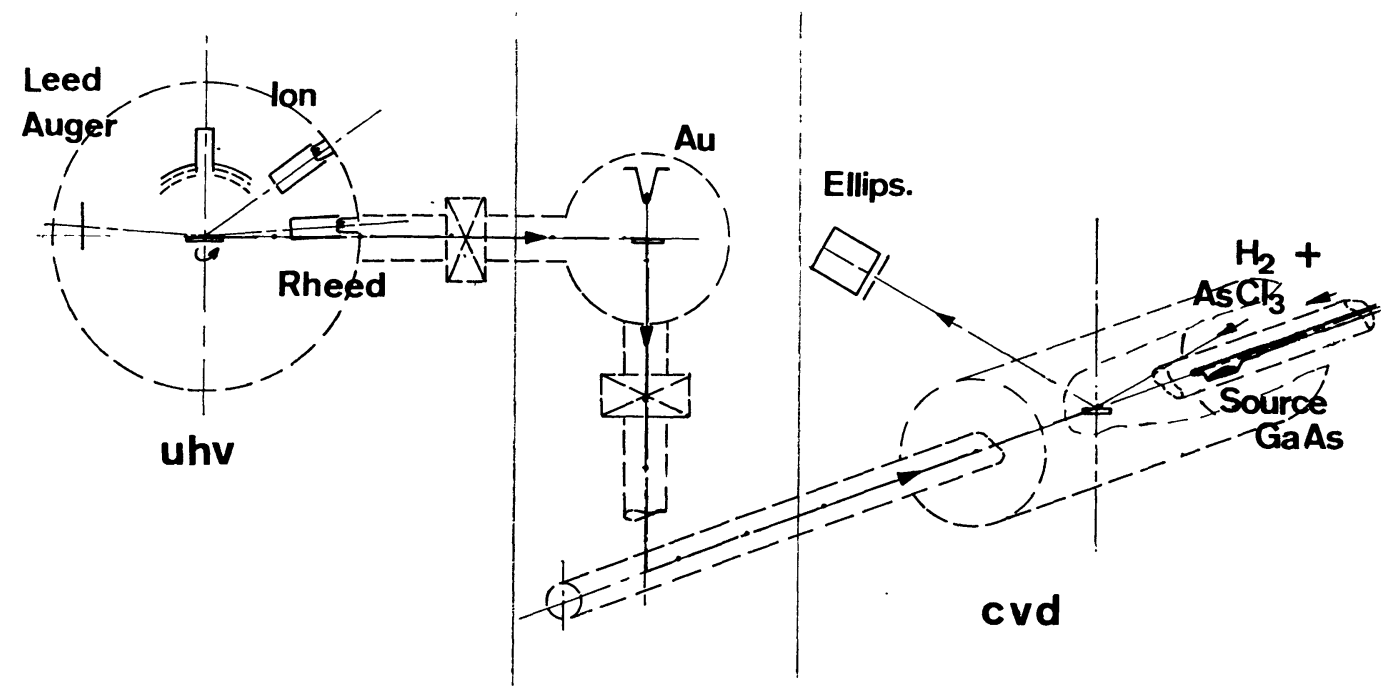

FIG. 1. - Vue générale du système réacteur CVD-sas-bâti ultra-vide. 
permettant l'entrée et la sortie du faisceau lumineux d'ellipsométrie sous une incidence de $72^{\circ}$. Le four est mobile : pour arrêter la croissance et effectuer le transfert, on retire le four de la région d'épitaxie. L'échantillon refroidit alors rapidement (passage de $750^{\circ} \mathrm{C}$ à $600^{\circ} \mathrm{C}$ en 30 secondes). On arrête en même temps l'arrivée de $\mathrm{AsCl}_{3}$ et le chauffage de la source afin de réaliser une trempe chimique et thermique de l'échantillon. Il est clair que le choix d'une procédure d'arrêt de croissance est un point capital pour le succès du transfert de l'échantillon. L'ellipsomètre permet d'étudier les effets de la trempe et de guider le choix de la bonne procédure.

2.2 SYSTÈME DE TRANSFERT. - La coexistence des ambiances d'épitaxie vapeur et d'ultra-vide impose des restrictions sévères sur le choix des matériaux. L'atmosphère du réacteur est très corrosive et seuls le quartz et le carbone vitreux peuvent être considérés comme inertes. Il est ainsi impossible d'envisager des prises de contact électrique sur l'échantillon (amenées de courant de chauffage, thermocouples). De même on ne peut pas fixer mécaniquement l'échantillon suivant les méthodes usuelles (ressorts, bandes de Mo, vis d'acier...). D'autre part, l'emploi des techniques LEED et RHEED impose d'éliminer tout isolant (le quartz en particulier) au voisinage de l'échantillon dans l'ultravide afin d'éviter les effets de charge qui déformeraient les figures de diffraction. Enfin, pour pouvoir être aussi proches que possible des conditions hydrodynamiques habituelles d'un réacteur C. V. D., nous avons gardé la géométrie d'un tube de quartz cylindrique pour l'échantillon en cours de croissance. La liaison avec le bâti-ultra vide nécessite donc des déplacements importants (trajet supérieur à $1 \mathrm{~m}$ ) qui entraînent l'emploi d'un sas intermédiaire avec son propre porte-échantillon. Pour satisfaire aux conditions ci-dessus, nous avons opté pour le système représenté sur la figure 2 .

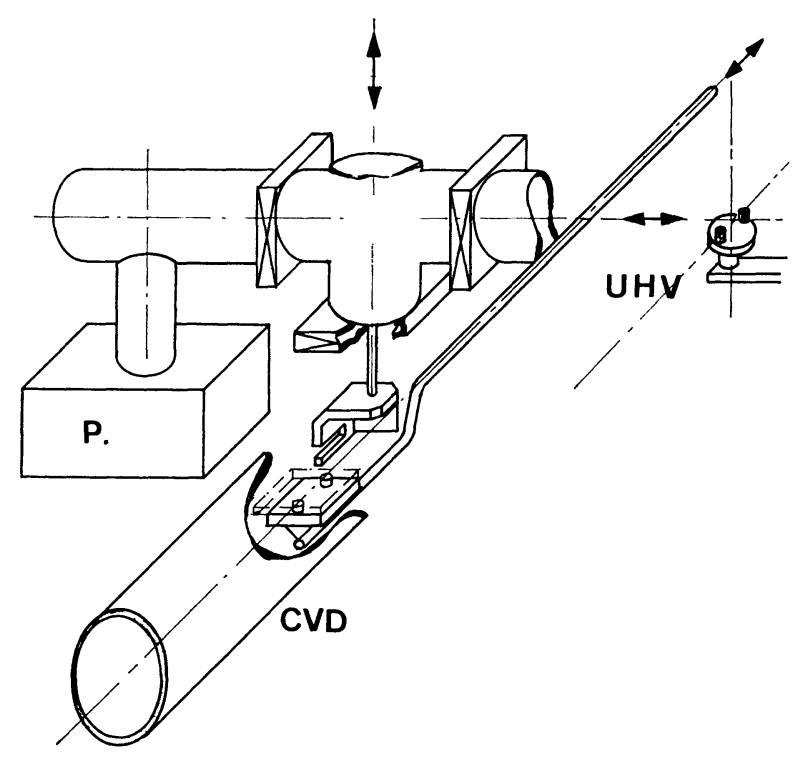

Fig. 2. - Vue détaillée du système de transfert.
L'échantillon, de dimensions typiques

$$
2 \mathrm{~mm} \times 10 \mathrm{~mm} \times 10 \mathrm{~mm},
$$

est posé sur une sole en carbone vitreux percée de 2 trous qui en permettent l'indexation. C'est cet ensemble échantillon + sole que l'on transfère. Dans le réacteur C. V. D., il est posé sur une palette de quartz muni de 2 pointes qui fixent la position. La palette est solidaire d'un tube de quartz manœuvrable de l'extérieur qui permet l'alignement de l'échantillon pour l'ellipsométrie et son déplacement vers le bâti ultra-vide.

L'étanchéité, lors de ce déplacement, est assurée par un joint Viton. On maintient en outre le réacteur C. V. D. en légère surpression d'hydrogène de manière à éviter toute contamination par les gaz atmosphériques. Les mesures de spectroscopie Auger montrent d'ailleurs que le taux de recouvrement en carbone (test particulièrement sensible d'un contact avec l'atmosphère) reste très faible pour une surface initialement propre en ultra-vide qu'on ramène dans le bâti après transfert dans le réacteur.

Le transfert entre réacteur et bâti se fait au moyen d'un sas muni de vannes-tiroirs (joints Viton). Le sas possède un système de pompage autonome :

- une pompe primaire à palettes avec tamis moléculaire et piège à azote liquide (comme il s'agit de pomper de l'hydrogène, les pompes à sorption sont inadéquates),

- une pompe à sublimation de titane avec paroi refroidie à l'azote liquide en série avec une pompe ionique $50 \mathrm{l} / \mathrm{s}$.

Dans cette chambre intermédiaire, la sole de carbone qui porte l'échantillon est maintenue par la pesanteur dans une pièce métallique fendue. Cette pièce se déplace verticalement et assure le relais entre les deux porteéchantillons horizontaux du réacteur et du bâti. Ce système, de par le faible volume du sas ( $1 \mathrm{dm}^{3}$ environ $)$, permet un passage rapide entre les deux ambiances.

2.3 BAti UlTRA-VIDE. - La disposition schématique d u montage ultra-vide est donnée figure 3. Pour rendre

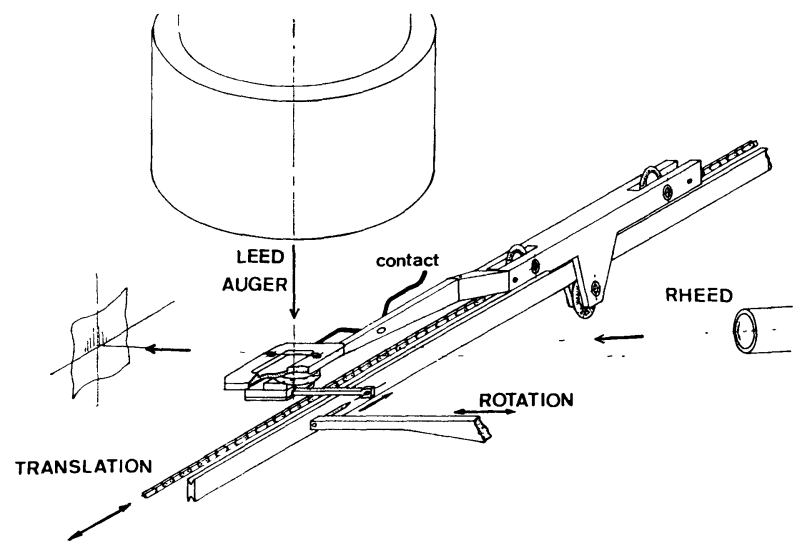

FIG. 3. - Disposition des éléments dans le bâti ultra-vide. 
le système de transfert aussi simple que possible, il a fallu réduire au minimum les mouvements et les prises de contact sur le porte-échantillon ultra-vide. Il s'agit d'un plateau horizontal muni de pointes pour indexer la sole de carbone. Ce plateau peut être translaté depuis le sas jusqu'à la position d'observation en LEED, RHEED, AUGER à l'aide d'une chaîne. Cette solution est en effet la seule qui permette une telle distance de translation $(400 \mathrm{~mm})$ sous un encombrement réduit. En position d'observation, une lame de cuivre solidaire du plateau vient au contact avec une amenée de courant. On peut ainsi polariser la sole en carbone vitreux par rapport à la masse. Cela permet de chauffer l'échantillon par bombardement électronique $(2 \mathrm{kV}$, $20 \mathrm{~mA}$ ). On atteint aisément $600^{\circ} \mathrm{C}$, température mesurée depuis l'extérieur à l'aide d'un pyromètre optique (pyromètre RAYTEX gamme $350^{\circ} \mathrm{C}-800^{\circ} \mathrm{C}$ ). On peut également faire un bombardement ionique pour décaper la surface $\left(10^{-4}\right.$ torr d'Argon, ions de $300 \mathrm{eV}, 10 \mu \mathrm{A}$ de courant cible).

La position d'observation est située au centre de l'ensemble grilles-écran hémisphérique d'un canon LEED VARIAN. Les mesures LEED et AUGER sont donc possibles sans mouvement supplémentaire de l'échantillon. Pour la diffraction RHEED, on peut faire tourner le plateau porte-échantillon autour d'un axe vertical. On obtient ainsi les diagrammes aux différents azimuts. Le réglage de l'angle d'incidence du faisceau RHEED (pour optimiser la longueur des barres du diagramme) est obtenu à l'aide des plaques déflectrices du canon RHEED et en polarisant l'échantillon.

3. Mesures in situ. - Pour illustrer le fonctionnement de l'appareillage nous donnerons dans ce qui suit une série de résultats typiques obtenus aux divers stades d'un processus d'épitaxie. Nous discuterons d'abord les mesures faites in situ, montrant ce qu'elles apportent mais aussi leurs limitations. Nous présenterons alors les mesures faites par transfert dans l'ultravide, montrant comment elles complètent et permettent de lever les ambiguïtés des mesures in situ.

3.1 DESCRIPTION DE L'ELLIPSOMÉTRIE. - 3.1.1 Conditions expérimentales. - L'ellipsométrie étant une technique pratiquée de longue date (les premières publications datent du siècle dernier), ses performances et ses divers aspects expérimentaux et théoriques ont été largement explorés. Une revue récente en a été faite lors d'une conférence aux U.S. A. en 1968 [8]. Les surfaces des semiconducteurs en particulier (Si [9] Ge [10] GaP [11]) ont fait l'objet d'analyses détaillées. Par rapport à un ellipsomètre idéal tel que le définissent ces publications, nos conditions expérimentales imposent des restrictions sévères :

1) modifier le moins possible l'environnement de l'échantillon en cours d'épitaxie,

2) pouvoir analyser des phénomènes rapidement variables comme les effets de décapage, de début de croissance, de trempe lors du transfert.

La restriction 1 nécessite de garder le tube de quartz cylindrique du réacteur autour de l'échantillon. Elle exclut l'emploi de fenêtres optiques planes et sans contraintes pour les faisceaux d'entrée et de sortie d'ellipsométrie.

La restriction 2 implique l'emploi d'un système automatique à acquisition rapide (jusqu'à plus de 20 mesures par seconde) et élimine les procédures habituelles d'annulation de signal par rotation manuelle des polariseurs.

L'appareillage réalisé est schématisé sur la figure 4. Il reprend, avec les modifications nécessaires, les éléments d'un ellipsomètre automatique déjà réalisé dans nos laboratoires [12]. Dans sa version actuelle il est proche du système mis au point par $\mathrm{P}$. S. Hauge et F. H. Hill [13].

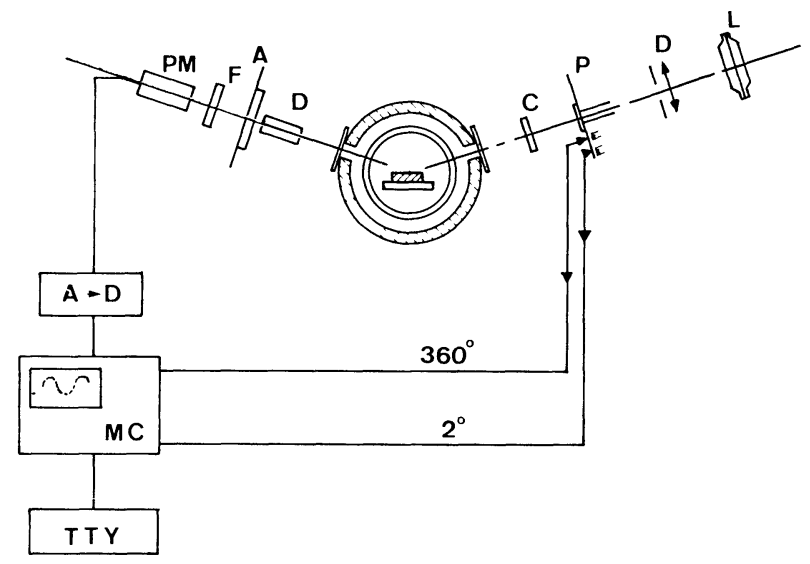

FIG. 4. - Ellipsomètre pour les mesures in situ et électronique associée. $\mathrm{L}=$ lampe à vapeur de mercure, $\mathrm{D}=$ diaphragmes définissant un faisceau de $\varnothing 1 \mathrm{~mm}, \mathbf{P}=$ polariseur, $\mathrm{A}=$ analyseur, $\mathrm{C}=$ compensateur (facultatif) (lame $5 \lambda / 4$ avec $\lambda=546 \mathrm{~nm}), \mathrm{F}=$ filtre interférentiel, $\mathrm{MC}=$ analyseur multicanal, TTY $=$ télétype ou interface ordinateur.

3.1.2 Partie optique. - La source utilisée est une lampe à vapeur de mercure haute pression type $\mathrm{HBO}$ $100 \mathrm{~W} / 2$. Le détecteur est un photomultiplicateur à réponse spectrale panchromatique type XP 1020. La sélection en longueur d'onde est faite à l'aide d'un filtre interférentiel. Pour augmenter le rapport signal/bruit, le filtre est placé directement à l'entrée du P. M. Ceci a l'avantage supplémentaire d'utiliser toute la lumière de la source tout le long du trajet dans le réacteur CVD ; le faisceau est plus visible et les alignements peuvent se faire à la lumière ambiante. Pour les résultats présentés ici, la longueur d'onde est fixée à $\lambda=5461 \AA$. On utilise une lame de quartz d'épaisseur $5 \lambda / 4$ comme compensateur. Le polariseur et l'analyseur sont des polariseurs polaroïd.

Comme nous l'avons indiqué ci-dessus, la partie délicate de ce système optique se situe au niveau du réacteur CVD. Pour minimiser les effets dus à la courbure du tube de quartz du réacteur (diamètre $40 \mathrm{~mm}$ ) 
on utilise un faisceau parallèle limité par des diaphragmes à un diamètre de $1 \mathrm{~mm}$ et l'échantillon est placé sur l'axe du réacteur. Le tube de quartz étant recuit à $1100^{\circ} \mathrm{C}$ et n'étant pas fixé rigidement à des supports métalliques, on peut le considérer comme sans contrainte, ce que confirme un examen entre polariseurs croisés.

Pour permettre le passage des faisceaux, le four entourant le réacteur est percé de 2 trous de diamètre $5 \mathrm{~mm}$. L'angle d'incidence $\theta$ du faisceau est donc fixé. On l'a choisi égal à $72^{\circ}$, valeur habituelle, pour les mesures sur $\mathrm{Si}$ et $\mathrm{GaAs}$ dont l'indice de réfraction est de l'ordre de 4 (angle de Brewster proche de $76^{\circ}$ ) L'existence de fenêtres dans le four entraîne une perte de calories d'autant plus néfaste qu'elle peut créer des points froids et donc des zones de dépôt sur le tube de quartz et ceci précisément aux endroits de passage du faisceau lumineux. Pour éviter ces points froids, on applique des lames de verre sur les fenêtres du four; les pertes par convection sont ainsi éliminées et le dépôt sur le tube ne se produit plus. L'inconvénient de cette méthode vis-à-vis de l'ellipsométrie est d'ajouter 4 interfaces supplémentaires sur le trajet des faisceaux. Les conditions expérimentales imposées par la restriction 1 ne permettent donc pas des mesures absolues d'ellipsométrie de par le nombre d'interfaces interposées sur le trajet du faisceau. En fait, nous avons seulement besoin de mesures relatives par rapport à des états standard reproductibles à volonté (par exemple, échantillon à température ambiante après nettoyage dans l'ultra-vide contrôlé par LEED, RHEED et AUGER) puisqu'il s'agit de détecter les modifications apportées à une surface par l'épitaxie en phase vapeur.

3.1.3 Electronique associée. - Si on désigne par $P$ et $A$ les angles que font les plans de polarisation du polariseur et de l'analyseur par rapport au plan d'incidence, si on place la lame quart d'onde comme indiqué sur la figure 4 avec ses axes lent et rapide à $45^{\circ}$ de part et d'autre du plan d'incidence, on montre facilement que l'intensité lumineuse reçue par le $\mathbf{P}$. M. est de la forme :

$$
I_{\mathrm{PM}} \propto\left[\operatorname{tg}^{2} A+\operatorname{tg}^{2} \psi+2 \operatorname{tg} A \operatorname{tg} \psi \sin (2 P-\Delta)\right]
$$

$\Delta$ et $\psi$ sont les angles ellipsométriques caractéristiques de l'échantillon analysé. Ils expriment les différences d'amplitude et de phase entre les facteurs de réflexion $R_{\|}$ et $R_{\perp}$ de la surface pour une lumière polarisée respectivement parallèlement et perpendiculairement au plan d'incidence ; on a la relation :

$$
\frac{R_{\|}}{R_{\perp}}=\operatorname{tg} \psi \cdot \exp (i \Delta) .
$$

On voit, d'après (3), que $I_{\mathrm{PM}}$ sera nul pour $A=\psi$ et $2 P=\Delta-90^{\circ}$. Ceci constitue le principe de la méthode classique [9] qui mesure $\Delta$ et $\psi$ en minimisant $I_{\mathrm{PM}}$ par variation manuelle de $A$ et $P$; cette procédure demaste plusieurs minutes pour une mesure de $\Delta$ et $\psi$ précise à $0,01^{\circ}$ près. La restriction 2 impose une cadence beaucoup plus rapide. On choisit alors de faire tourner le polariseur. Le signal reçu sur le P. M. est alors un signal sinusoïdal dont on peut noter les extrêmes $I_{\max }$ et $I_{\min }$ ainsi que la phase $\varphi$ de passage à la valeur moyenne. On a ainsi :

$$
\begin{aligned}
& \Delta=2 \varphi \\
& \psi=\operatorname{arctg} \frac{r-1}{r+1} \text { avec } r=\left(\frac{I_{\max }}{I_{\min }}\right)^{1 / 2}
\end{aligned}
$$

si on fixe la position de l'analyseur de telle façon que $A=45^{\circ}$. Remarquons que cette méthode, utilisant une lame quart d'onde, n'est valable qu'à une longueur d'onde donnée. Si on veut faire une étude en fonction de la longueur d'onde, il faut utiliser un compensateur différent pour chaque longueur d'onde. Une solution plus simple consiste à supprimer le compensateur. En gardant $A=45^{\circ}$ on montre [13] que l'intensité reçue par le P. M. est de la forme

$I_{\mathrm{PM}}^{\prime} \propto[1-\cos 2 \psi \cdot \cos 2 P+\sin 2 \psi \cos \Delta \cdot \sin 2 P]$.

On peut ainsi évaluer numériquement [13], à partir de mesures échantillonnées de $I_{\mathrm{PM}}^{\prime}$, les coefficients du développement de Fourier :

$$
I_{\mathrm{PM}}^{\prime} \propto[1+a \cos 2 P+b \sin 2 P]
$$

on a alors

$$
\begin{aligned}
& \psi=\frac{1}{2} \arccos (-a) \\
& \Delta=\arccos \left(\frac{b}{\sqrt{1-a^{2}}}\right) .
\end{aligned}
$$

Ainsi, en modifiant seulement le filtre interférentiel placé devant le P. M., on obtient l'évolution de $\Delta$ et $\psi$ en fonction de $\lambda$.

Dans le système que nous avons réalisé (Fig. 4), le polariseur est monté sur un moteur à arbre creux que l'on fait tourner à environ 30 tours/seconde. Le disque tournant sur lequel est monté le polariseur est pourvu d'une couronne de 180 dents régulièrement espacées. Ces dents défilent devant un système lampe-photodiode. Quand le disque tourne on obtient ainsi un top à chaque fois qu'une dent obture le faisceau de la lampe, ce qui fournit un repérage de la position angulaire du disque tous les $2^{\circ}$. On utilise ces tops $2^{\circ}$ comme ordres d'échantillonnage du signal issu du P. M. par un codeur analogique-digital de haute précision (8 192 niveaux de digitalisation), organe d'entrée d'un analyseur multicanal S. E. I. N. type ZOOMAX. Les tops $2^{\circ}$ servent à la commande d'avance adresse. Un trou supplémentaire percé dans le disque tournant permet d'obtenir un top à chaque rotation. Ce top $360^{\circ}$ est utilisé comme origine et sert à la commande de départ acquisition. A chaque rotation du disque, on obtient l'échantillonnage tous les $2^{\circ}$ de deux sinusoïdes de $I_{\mathrm{PM}}$, chaque échantillon étant mesuré à $1 / 8192$ près. 
Remarquons que l'échantillonnage étant relié directement à la position angulaire du disque, l'acquisition est indépendante de la vitesse de rotation qui n'a donc pas besoin d'être régulée. La vitesse maximale d'acquisition est limitée par la cadence imposée par le codeur $(180 \mu \mathrm{s}$ minimum par échantillonnage) et correspond à environ 60 sinusoïdes par seconde. On peut ainsi effectuer jusqu'à 60 mesures par seconde. Si on n'a pas besoin d'une telle cadence, on peut accumuler le signal sur $N$ sinusoïdes, améliorant ainsi le rapport signal/bruit d'un facteur $\sqrt{N}$. Après acquisition le signal est exploité numériquement (en temps différé) et comparé, par les méthodes de moindres carrés, aux formules (3) ou (8) suivant qu'on utilise ou non un compensateur. La précision finale sur $\Delta$ et $\psi$ (dispersion statistique d'une série de mesures faites sans modifier ni les positions des polariseurs, ni l'état de surface) est de l'ordre de $0,01^{\circ}$ pour $N=16$. La reproductibilité (dispersion statistique d'une série de mesures faites sur un même état de surface, après avoir modifié les positions d'échantillon et de polariseurs puis refait les alignements) est de l'ordre de $0,1^{\circ}$. Quant l'alignement est réalisé, l'erreur due aux fenêtres n'excède pas $0,1^{\circ}$.

3.2 RÉSultats TYPIQUeS. - 3.2.1 Effet de la température. - Le processus de l'épitaxie phase vapeur comporte un paramètre soigneusement maintenu constant dans l'ellipsométrie classique : la température. Ceci entraîne une difficulté supplémentaire dans l'interprétation: les angles $\Delta$ et $\psi$ peuvent varier, quand $T$ varie, sans que l'échantillon se modifie. On sait en effet que l'indice de réfraction $\tilde{n}$ d'un milieu varie avec $T$. Toute variation de $\tilde{n}$ entraînant une variation des angles $\Delta$ et $\psi$ il conviendra donc de reconnaître dans les $\delta \Delta$ et $\delta \psi$ mesurés la contribution $(\delta \Delta)_{0}$ et $(\delta \psi)_{0}$ due à cet effet, les différences $\delta \Delta-(\delta \Delta)_{0}$ et $\delta \psi-(\delta \psi)_{0}$ étant seules imputables à des changements chimiques ou de structures de l'échantillon et de sa surface. La figure 5 illustre ce point de vue. Sur la figure $5 a$, on a représenté $\delta \psi$ mesuré en fonction de $T$ sur un échantillon de GaAs (100) nettoyé dans l'ultra-vide puis soumis à plusieurs cycles de chauffage refroidissement dans le réacteur CVD sous atmosphère de $\mathrm{H}_{2}$ (régime de montée en température décrit au paragraphe 1). On constate qu'un nouveau cycle de variation de $T$ provoque des variations $\delta \psi$ sensiblement identiques à $T$ croissant et décroissant. On peut admettre alors que la surface est dans un état stable et que l'on mesure $(\delta \psi)_{0}$. On constate que $(\delta \psi)_{0}$ est pratiquement proportionnel à $T$. Ce fait a été vérifié de nombreuses fois sur GaAs et $\mathrm{Si}$. $(\delta \psi)_{0}$ constitue en fait un indicateur de la température du substrat.

Sur la figure $5 b$ on a représenté au contraire les variations de $\delta \psi$ en fonction de $T$ pour un échantillon de GaAs (100) préalablement bombardé avec des ions Argon dans l'ultra-vide de façon à perturber fortement le réseau cristallin au voisinage de la surface.

On constate un hystérésis dans le cycle de $\delta \psi$ en fonction de $T$ indiquant une modification de l'échan-
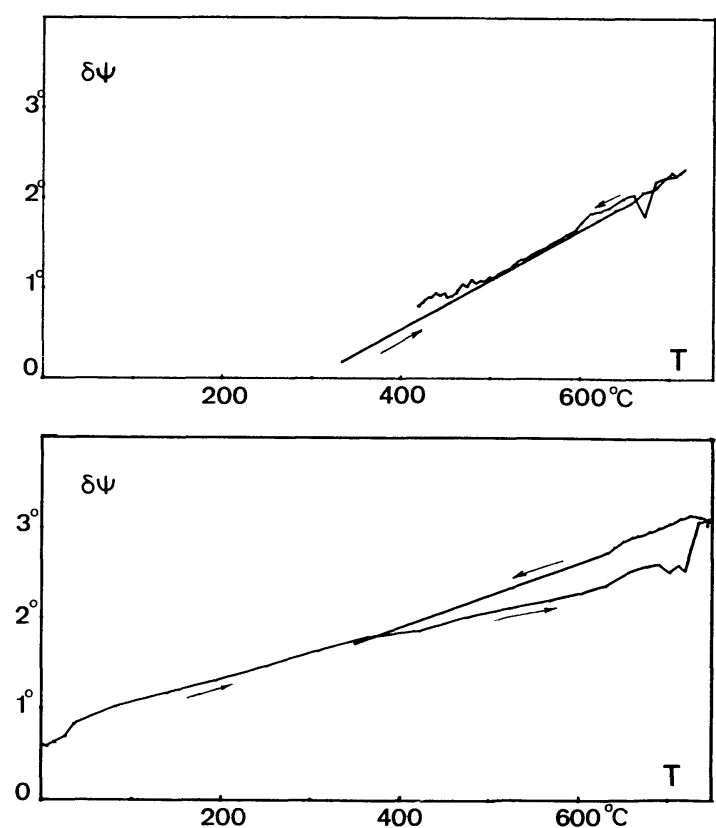

Fig. 5. - Variation de $\psi$ en fonction de la température pour des échantillons de $\mathrm{GaAs}$ chauffés sous atmosphère d'hydrogène dans le réacteur CVD $\left(\lambda=546 \mathrm{~nm}\right.$, incidence $\left.72^{\circ}\right)$. a) Echantillon ayant déjà subi plusieurs cycles de chauffage à $750^{\circ} \mathrm{C}$ et refroidissement. $b$ ) Echantillon venant de l'ultra-vide dont la surface a été volontairement perturbée par un bombardement ionique.

tillon : les défauts provoqués par le bombardement ionique sont recuits. D'après l'évolution brusque de $\delta \psi$ autour de $700^{\circ} \mathrm{C}$ on peut penser que le recuit est très efficace aux alentours de cette valeur de $T$. Au refroidissement $\delta \psi$ varie linéairement avec $T$, avec une pente cependant plus faible que sur la figure $5 a$. On peut penser à un recuit imparfait des défauts.

Ces résultats montrent l'apport de l'ellipsométrie dans les régimes à température variable. On notera toutefois la valeur importante de $(\delta \psi)_{0}$ (environ $3^{\circ}$ quand $T$ varie de 25 à $750^{\circ} \mathrm{C}$ ) ce qui rend difficile une estimation précise de $\delta \psi-(\delta \psi)_{0}$ qui contient les informations sur les changements subis par l'échantillon. D'autre part, quand $T$ varie dans une telle gamme de température, les dilatations provoquent inévitablement de légers déplacements du porteéchantillon, des contraintes dans les fenêtres... Ceci est la cause des variations erratiques de $\delta \psi$ observables sur la figure 5, variations qui peuvent être de l'ordre de $0,1^{\circ}$. En conclusion, à température variable, l'ellipsométrie permet seulement de détecter les changements structuraux et chimiques importants.

3.2.2 Effets des gaz d'épitaxie en régime isotherme. - Si on maintient $T$ constant, les causes d'erreur mentionnées ci-dessus disparaissent et on peut à nouveau obtenir une précision de $0,01^{\circ}$ sur $\Delta$ et $\psi$. Ainsi les régimes de décapage et de croissance définis au paragraphe 1, étant isothermes, sont mesurables avec une très bonne précision. Nous reportons sur la figure 6 deux exemples typiques d'évolution de l'échantillon au cours de ces régimes. Sur la figure $6 a$, on a représenté 

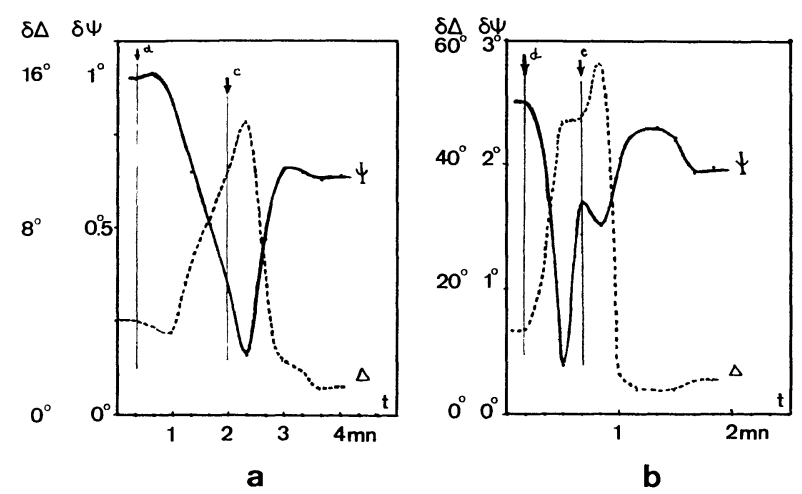

Fig. 6. - Effets sur les angles $\Delta$ et $\psi$ d'ellipsométrie d'un décapage suivi d'une croissance $\left(\lambda=546 \mathrm{~nm}\right.$, incidence $\left.72^{\circ}\right)$. $a$ ) Décapage très dilué et long. $b$ ) Décapage concentré et court. On notera les différences d'échelles entre les cas $a$ ) et $b$ ).

$\delta \Delta$ et $\delta \psi$ correspondants au processus chronologique suivant :

1) Avant $t=d$ l'échantillon est à $750^{\circ} \mathrm{C}$ sous $\mathrm{H}_{2}$. $\Delta$ et $\psi$ sont constants, on a un état stable. Notons que cette stabilité est toujours observée et ne dépend pas de l'histoire de l'échantillon.

2) A l'instant $t=d$ on commence le régime de décapage défini au paragraphe 1 , mais avec un flux 2 fois plus faible de $\mathrm{AsCl}_{3}$ et on coupe au bout de $5 \mathrm{~s}$ la circulation de $\mathrm{H}_{2}$ sur $\mathrm{AsCl}_{3}$. On réalise ainsi un décapage très dilué. $\Delta$ et $\psi$ évoluent continûment, même après l'arrêt du flux de $\mathrm{AsCl}_{3}$ indiquant une évolution continuelle de la surface.

3) A l'instant $t=c$ soit $100 \mathrm{~s}$ après $t=d$, on commence le régime de croissance défini au paragraphe 1 .

$\Delta$ et $\psi$ varient alors rapidement pour se stabiliser après $100 \mathrm{~s}$ environ. On atteint alors un état stable, celui de l'interface gaz-solide en cours de croissance.

Sur la figure $6 b$, on a représenté $\delta \Delta$ et $\delta \psi$ dans le cas d'un processus plus rapide :

1) Avant $t=d$, on est dans un état stable sous $\mathrm{H}_{2}$ à $750{ }^{\circ} \mathrm{C}$.

2) A $t=d$, on commence le décapage défini au paragraphe 1 .

Les variations $\delta \Delta$ et $\delta \psi$ ont les mêmes tendances que dans le cas $6 a$ mais sont rapides et plus fortes. Au bout de $20 \mathrm{~s}, \delta \psi$ change de sens de variation ce qui indique une modification de la façon dont l'échantillon se décape.

3) A $t=c=d+30 \mathrm{~s}$, on arrête le décapage et on commence la croissance définie au paragraphe 1 . A nouveau $\delta \Delta$ et $\delta \psi$ ont des allures similiares au cas $6 a$ et on atteint l'état stable de croissance après $60 \mathrm{~s}$ environ.

On peut constater que l'état stable sous $\mathrm{H}_{2}$ à $750^{\circ} \mathrm{C}$, et l'état stable de croissance sont assez proches en valeur de $\Delta$ et $\psi$. Le décapage a des effets très violents (jusqu'à $50^{\circ}$ pour $\Delta$ en moins de $60 \mathrm{~s}$ !) et partant assez incontrôlables. Nous montrerons au paragraphe suivant ses effets néfastes sur la rugosité de la surface. Il est d'ailleurs bien connu que cette étape du processus d'épitaxie est délicate et aboutit à une destruction irréversible de l'échantillon quand on n'optimise pas les flux et les pressions partielles. Comme le montre la figure 6 , le processus de croissance restaure les défauts provoqués par le décapage et aboutit à un état acceptable de l'interface gaz-solide en une minute environ. On voit tout l'intérêt que présente l'ellipsométrie dans le contrôle et l'optimisation de l'enchaînement décapage-croissance.

L'intérêt et la sensibilité de la méthode d'ellipsométrie est donc bien établie. Mais, au vu des résultats des figures 5 et 6 , il est clair que l'interprétation en données structurales et chimiques des divers états repérés par cette méthode passe par une analyse complémentaire en ultra-vide.

4. Mesures en ultra-vide. - L'analyse détaillée des résultats LEED, RHEED et AUGER obtenus aux divers stades du processus d'épitaxie dépasse le cadre de cet article. L'étude en particulier de l'état stable sous $\mathrm{H}_{2}$ à $750^{\circ} \mathrm{C}$, pour $\mathrm{GaAs}(100)$ et les surfaces vicinales à (100) fait l'objet d'autres publications [14] : on a pu notamment montrer le rôle du chlore dans cet état stable (formation d'une couche ordonnée de structure $(1 \times 2)$ sur GaAs $(100))$ et examiner également la stabilité des systèmes de marches monoatomiques sur les surfaces vicinales en fonction de la désorientation. Nous discuterons ici de trois états de la surface d'épitaxie : à l'introduction dans le réacteur, après un décapage, après une croissance de quelques $\mu \mathrm{m}$ de la couche épitaxiale. Les diagrammes RHEED correspondant sont rassemblés sur la figure 7 .

La figure $7 a$ présente le diagramme RHEED d'un échantillon de GaAs (001) venant de l'extérieur et préparé par les méthodes usuelles de polissage mécanochimique. L'aspect macroscopique de la surface est celui d'un très bon miroir.

Le diagramme RHEED est formé de taches légèrement allongées ce qui indique une rugosité assez forte de la surface. Le faible allongement des taches indiquent que les dimensions des parties atomiquement planes d'orientation (100) n'excèdent pas $20 \AA$ en moyenne. La diffraction LEED ne fournit d'ailleurs aucun diagramme ce qui confirme l'absence d'ordre cristallin sur des distances inférieures ou égales au diamètre de cohérence LEED (quelques centaines d'Å). L'analyse AUGER indique la présence d'oxygène, de carbone et de chlore (venant de l'atmosphère du réacteur).

$\mathrm{Si}$ on nettoie alors la surface par bombardement ionique et recuit à $600^{\circ} \mathrm{C}$, on obtient le diagramme RHEED de la figure $7 b$, caractéristique de la structure $\mathrm{C}(8 \times 2)$ de la surface propre de GaAs (001) [15]. Le diagramme est alors formé de barres allongées indiquant une très bonne spécularité de la surface. Le diagramme LEED est bien celui d'une structure $C(8 \times 2)$. L'analyse AUGER ne révèle que les transitions caractéristiques de $\mathrm{Ga}$ et As. 


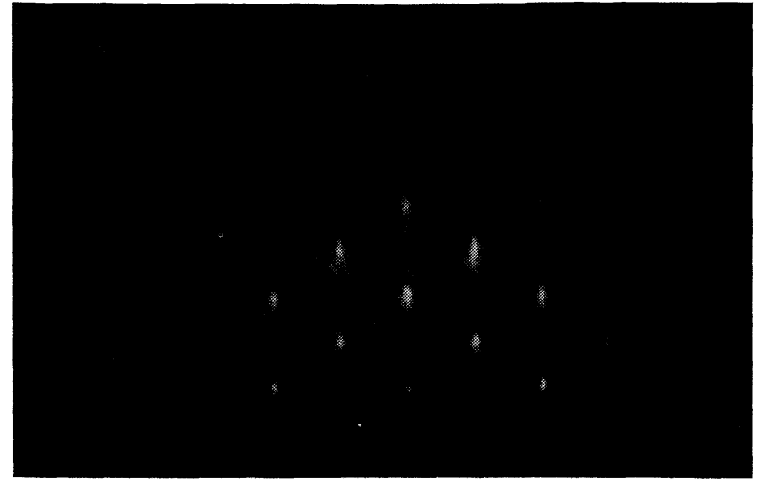

(a)

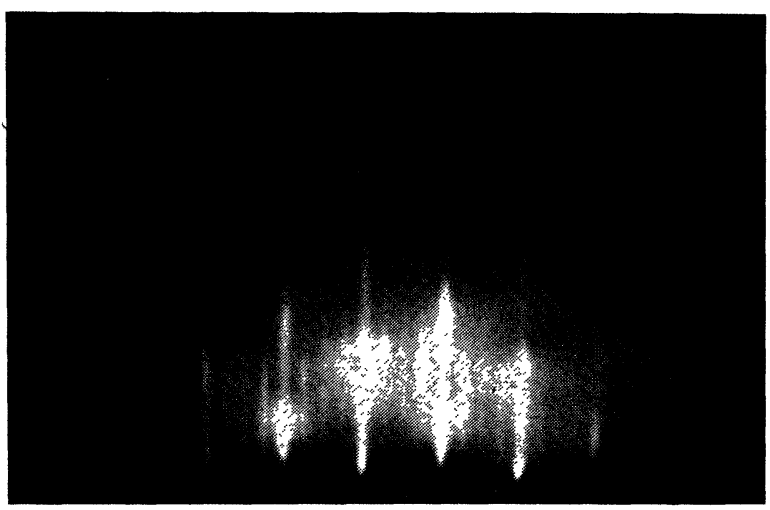

(b)

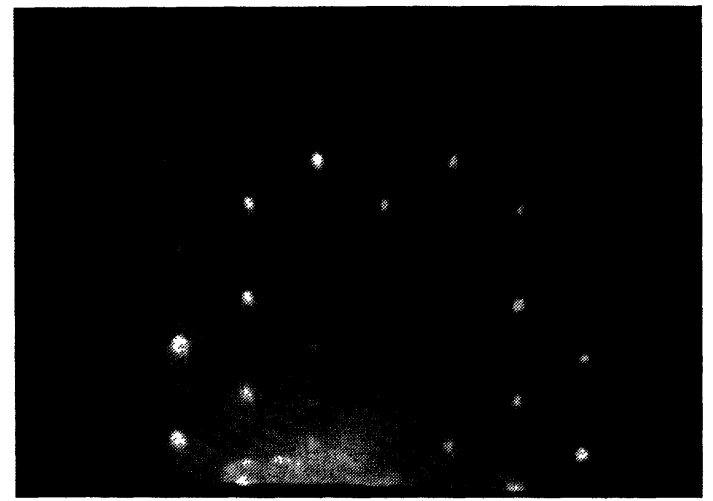

(c)

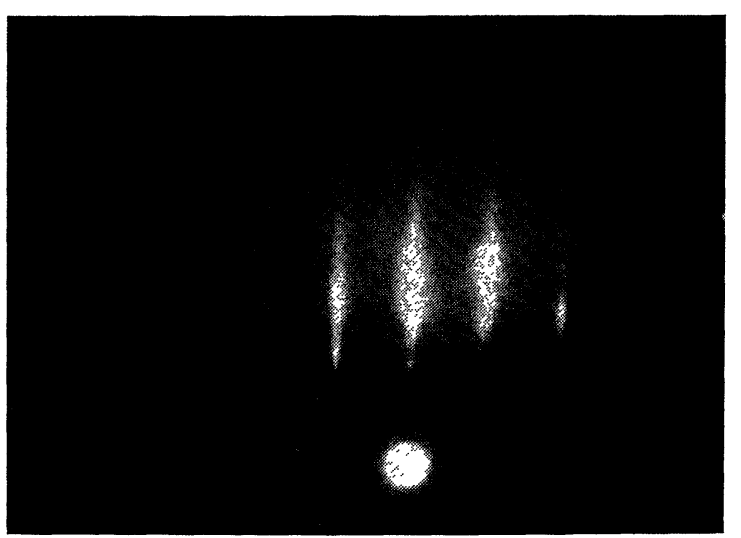

(d)

FIG. 7. - Diagrammes RHEED typiques sur GaAs (001) azimut [110].a) Venant de l'atmosphère, après polissage mécanochimique. b) Après nettoyage dans l'ultra-vide. c) Après un décapage dans le réacteur CVD. $d$ ) Après quelques minutes d'épitaxie.
Si on porte alors cette surface dans le réacteur et qu'on effectue la mise en température et le décapage arrêtant le processus entre les instants $t=d$ et $t=c$ de la figure 6 pour retransférer dans l'ultra-vide, on obtient le diagramme RHEED de la figure $7 c$. Le diagramme est à nouveau formé de taches mais elles ne sont plus allongées comme pour $7 a$. On a une surface très rugueuse, ne comportant plus de parties atomiquement planes d'orientation (100). Ceci confirme les effets néfastes du décapage sur la spécularité de la surface. Une étude détaillée en AUGER permettrait de déterminer la stœchiométrie d'une telle surface.

$\mathrm{Si}$, par contre, partant du même état de surface propre (Fig. 7b), on n'arrête le processus d'épitaxie que plusieurs minutes après $t=c$, c'est-à-dire après plusieurs $\mu \mathrm{m}$ de couche épitaxiale, le diagramme RHEED obtenu après transfert est celui de la figure $7 d$.

On constate qu'on obtient un diagramme de barres presque aussi allongées que pour $7 b$. On a donc toujours des portions de surfaces de dimensions au moins égales à $100 \AA$ qui sont des faces $(001)$ exactes. On n'observe plus de reconstruction de type $\mathrm{C}(8 \times 2)$ mais l'analyse AUGER indique la présence de chlore qui inhibe cette reconstruction. Le diagramme LEED est également un diagramme $(1 \times 1)$ aux points très bien définis. Dans les deux cas LEED et RHEED, on observe en outre un fond continu important, fond continu qui augmente avec la durée de la phase de croissance. Ceci indique un désordre à grande distance (à l'échelle de $1 \mu \mathrm{m}$ ) sur la surface. L'examen au microscope optique confirme cette observation: la surface présente des «vaguelettes » caractéristiques.

Remarquons que le fait d'obtenir des diagrammes LEED et RHEED sur une couche épitaxiale après transfert est une preuve très encourageante $\mathrm{du}$ bon fonctionnement de l'appareillage et de son adéquation au problème posé. On sait en effet l'extrême sensibilité de ces méthodes au désordre cristallin et à la contamination, la présence d'une ou deux monocouches désordonnées adsorbées suffisent généralement pour faire disparaître les diagrammes. D'autre part, l'absence de taches sur le diagramme RHEED $7 d$ de la couche épitaxiale indique que la croissance s'effectue bien couche après couche et non pas par germes tridimensionnels.

5. Conclusion. - L'appareillage présenté ci-dessus fonctionne donc de façon satisfaisante et les résultats déjà obtenus sur $\mathrm{GaAs}(100)$ montrent que la méthodologie adoptée est correcte. Nous n'avons pas cherché ici à démontrer que le transfert ne perturbait pas du tout l'objet d'étude; nous nous sommes limités à des exemples où des modifications mineures induites par le transfert ne remettent pas en cause les conclusions (par exemple la rugosité induite par le décapage). Une étude plus complète est en cours. La comparaison entre les mesures ultra-vide et l'ellipsométrie devrait permettre une interprétation détaillée des mesures 
in situ et apporter ainsi des indications précieuses sur les mécanismes de l'épitaxie. Il est clair que cette technique de mesure n'est pas limitée à GaAs et que toute croissance cristalline en phase vapeur est susceptible d'être analysée ainsi.
Remerciements. - Nous voudrions exprimer ici notre gratitude à toute l'équipe de $\mathrm{M}$. Hollan et plus particulièrement MM. Chane et Hallais pour leur aide dans la mise au point du réacteur CVD et les discussions fructueuses que nous avons eues avec eux.

\section{Bibliographie}

[1] (On trouvera une compilation récente de ces données dans) GrÜNBAUM, E., chap. 9 in Epitaxial growth, Matthews, J. W. (éditeur Academic Press New York) 1975.

[2] Voir la liste de références de l'article de Joyce, B. A. et Foxon, C. T., J. Cryst. Growth 31 (1975) 122.

[3] Ludeke, R., Chang, L. L., Esaki, L., Appl. Phys. Lett. 23,4 (1973) 201

[4] Shaw, D. W., J. Cryst. Growth 31 (1973) 130.

[5] CADoret, R. et CAdoret, M., J. Cryst. Growth 31 (1975) 142.

[6] Sur les problèmes de transport en phase vapeur on pourra consulter l'ouvrage récent de

FAKTOR, M. M. et GARRETT, J., Growth of Crystals from the Vapour.

[7] BAN, V. S., J. Electrochem. Soc. 118 (1971) 1473 ;

- J. Electrochem. Soc. 119 (1972) 761 ;

- J. Phys. Chem. Solids 34 (1973) 1119.
[8] Recent developments in Ellipsometry (Eds Bashara N. M.) Buckman. A. B. et Hall, A. C., Surf. Sci. 16 (1969).

[9] Bootsma, G. A., Meyer, F., Surf. Sci. 14 (1969) 52.

[10] Meyer, F., de KluizenaAr, E. E., Bootsma, G. A., Surf. Sci. 27 (1971) 88.

[11] Morgan, A. E., Surf. Sci. 43 (1974) 150.

[12] Boccon Gibod, D., Lebrun, J., Sevin, P., Contrat DGRST $\mathrm{n}^{\circ}$ 65.00.244.00.212.75.01, rapport final octobre 1967, BACH, P., rapport LEP B. 236, août 1972.

[13] Hauge, P. S., Hill, F. H., IBM J. Res. Develop. novembre 1973.

[14] Theeten, J. B., Hottier, F. à paraître. Hotrier, F., Thèse Doctorat $3^{\mathrm{e}}$ cycle, Université de ParisSud Orsay, mars 1976

[15] JonA, F., IBM J. Res. Develop. 9 (1965) 375. Сно, A. Y., J. Appl. Phys. 42 (1971) 2074.

[16] Hollan, L., Schiller, C., J. Cryst. Growth 22 (1974) 175. 